\title{
Review Article \\ The Gut Microbiota and Respiratory Diseases: New Evidence
}

\author{
Li Chunxi $\mathbb{D},{ }^{1}$ Liu Haiyue, ${ }^{2}$ Lin Yanxia, ${ }^{1}$ Pan Jianbing, ${ }^{1}$ and Su Jin $\mathbb{D}^{1}$ \\ ${ }^{1}$ Department of Respiratory and Critical Care Medicine, Nanfang Hospital, Southern Medical University, \\ Guangdong Guangzhou 510515, China \\ ${ }^{2}$ Department of Laboratory Medicine, Zhujiang Hospital, Southern Medical University, Guangdong Guangzhou 510282, China
}

Correspondence should be addressed to Su Jin; drsujin@126.com

Received 24 March 2020; Revised 13 June 2020; Accepted 18 June 2020; Published 31 July 2020

Academic Editor: Carlo Perricone

Copyright (C) 2020 Li Chunxi et al. This is an open access article distributed under the Creative Commons Attribution License, which permits unrestricted use, distribution, and reproduction in any medium, provided the original work is properly cited.

\begin{abstract}
Human body surfaces, such as the skin, intestines, and respiratory and urogenital tracts, are colonized by a large number of microorganisms, including bacteria, fungi, and viruses, with the gut being the most densely and extensively colonized organ. The microbiome plays an essential role in immune system development and tissue homeostasis. Gut microbiota dysbiosis not only modulates the immune responses of the gastrointestinal (GI) tract but also impacts the immunity of distal organs, such as the lung, further affecting lung health and respiratory diseases. Here, we review the recent evidence of the correlations and underlying mechanisms of the relationship between the gut microbiota and common respiratory diseases, including asthma, chronic obstructive pulmonary disease (COPD), cystic fibrosis (CF), lung cancer, and respiratory infection, and probiotic development as a therapeutic intervention for these diseases.
\end{abstract}

\section{Introduction}

Chronic respiratory diseases, including asthma and chronic obstructive pulmonary disease (COPD), as well as respiratory virus infection, are often accompanied by gastrointestinal diseases or symptoms [1-3]. Patients with gastrointestinal diseases, such as inflammatory bowel disease (IBD) and gastroesophageal reflux, are prone to develop pulmonary dysfunction and have an increased incidence of respiratory disease $[4,5]$. These connections suggest a vital communication between the gut and lung. The human microbiome is believed to contribute to homeostasis and disease and is responsible for the interactions between these two mucosal sites. Changes in microbial composition or/and diversity will not only directly affect the colonized organ itself but also impact distant organs and systems [6]. In particular, gut microbiota dysbiosis is associated with various diseases, such as allergies, autoimmune diseases, diabetes, obesity, and cancer [7]. Recently, an increasing amount of evidence has indicated that the gut microbiota is closely related to respiratory health and disease, playing a crucial role in the development of asthma, COPD, cystic fibrosis (CF), lung cancer, and respiratory infection [8-11]. In this review, we summarize the recent findings involving the relationship and mechanisms underlying the relationship between the gut microbiota and common respiratory diseases, including asthma, COPD, $\mathrm{CF}$, lung cancer, respiratory infection, and other respiratory diseases, and the use of probiotics for improving or treating these diseases.

\section{The Gut and Airway Microbiome}

The human gastrointestinal (GI) tract harbors approximately $10^{14}$ bacteria consisting up to 1000 different species [12], and the advance of sequencing technology has rendered the gut microbiota the most widely studied microbiome of the human body. However, the study of the airway microbiota is still in its infancy when compared with that of the gut microbiota. At the phylum level, Firmicutes and Bacteroidetes account for more than $90 \%$ of the gut microbial community [13]. The microbiota of the upper and lower respiratory tracts are distinct, with more Firmicutes and Actinobacteria in the nostril and more Firmicutes, Proteobacteria, and Bacteroidetes in the oropharynx [14], whereas there are more Bacteroidetes and Firmicutes in the lung [15]. At the genus level, Bacteroides, Faecalibacterium, and 
Bifidobacterium are enriched in the gut [16], while Prevotella, Veillonella, and Streptococcus are the prominent genera in the lung [15]. Although the gut and respiratory microbiota exhibit compositional differences, the epithelia of both the GI and respiratory tracts develop from a common embryonic structure, the anatomical structures and functions of the two mucosal sites are similar, and early-life microbial colonization of the gut and lung exhibits similarities. Therefore, accumulating evidence has highlighted the relationship and crosstalk between the gut and lung, referred to as the gutlung axis $[10,17,18]$.

The gut microbiota is affected by many factors, such as drugs, diet, mode of delivery, and feeding practices, which may play a role in susceptibility to respiratory diseases (Figure 1). For example, early-life acid-suppressive medications and antibiotic use, fast food consumption, caesariansection delivery, and formula feeding are correlated with an increased risk of asthma, while a higher fiber intake, vaginal delivery, and breastfeeding are negatively correlated with asthma [19-25]. In addition, diet, smoke, and drugs, such as antibiotic and immunosuppressant, can also influence rheumatoid arthritis (RA) and the gut microbiota [26, 27].

\section{The Gut Microbiota and Respiratory Diseases}

3.1. Asthma. Asthma is a chronic airway inflammatory disease characterized by reversible airflow restriction and airway hyperresponsiveness. The increasing morbidity and mortality of asthma have made it a serious threat to human health. According to the "hygiene hypothesis," early-life exposure to specific microbiota constituents is essential for the development and maturity of the immune system, and their absence may increase the susceptibility to asthma and allergic diseases [28]. With the advance of sequencing technologies, an increasing number of studies have revealed a close relationship between the gut microbiota and asthma. The gut microbiota is different between healthy controls (HCs) and asthmatic individuals and is associated with the development of asthma.

Higher microbial diversity is often regarded as beneficial. A recent study showed a connection between low gut microbial diversity in early life and asthma in childhood [29]. Breastfeeding may protect against asthma and allergic disease in children, and gut bacterial diversity was lower in formulafed infants than in breastfed infants [30]. In addition to microbial diversity, specific gut bacteria have also been found to be closely related to asthma. For instance, Clostridium and Eggerthella lenta were more abundant in the gut of asthma patients than in that of HCs [31]. Furthermore, the decrease in Bifidobacterium, Akkermansia, and Faecalibacterium abundances and the increase in Candida and Rhodotorula abundances increased a child's risk of developing allergies and asthma [32], and intestinal colonization by Clostridium difficile at 1 month of age was associated with asthma at 6-7 years of age [33]. Therefore, we speculate that the wellbalanced commensal microbiota in the GI tract may be beneficial to host health and that reduced microbial diversity may be a marker for underlying pathologic conditions.
Noting the altered gut microbiota in asthma patients, researchers have attempted to modulate the pulmonary immune response as well as prevent and treat asthma through improving the gut microbiota. Arrieta et al. revealed a significant reduction in Lachnospira, Veillonella, Faecalibacterium, and Rothia abundances in the gut of asthmatic infants. Furthermore, inoculations of these bacteria in germ-free (GF) mice ameliorated airway inflammation and prevented asthma development [34]. Similar results were obtained in both murine and human studies in which oral administration of Lactobacillus rhamnosus, Lactobacillus casei, and Bifidobacterium breve potentially prevented and treated allergies and asthma [35-37]. Another study indicated that probiotic intervention for pregnant women and their infants who were at a high risk of allergy could protect caesarian-delivered children from allergic disease [38]. Additionally, a double-blind, randomized, placebo-controlled trial of 160 asthmatic children suggested that Lactobacillus can reduce asthma severity and improve asthma control [39].

However, other studies have drawn opposite conclusions, finding that probiotics do not have significant benefits in asthmatic children [40]. In these randomized controlled trials (RCTs), probiotic supplementation (such as with Lactobacillus and Bifidobacterium) for 7 weeks to 6 months was found to have no preventive or therapeutic effects on children with a high risk of asthma or on asthmatic patients. No significant differences in the outcome measures were observed between the probiotic and placebo groups, including the incidence of asthma, clinical outcomes (asthmarelated events, quality of life, respiratory tract infections, antibiotic use, and asthma exacerbations), and pulmonary function (fraction of exhaled nitric oxide (FeNO) and forced expiratory volume in $1 \mathrm{~s}$ (FEV1)) [41-46]. Although probiotics had no significantly beneficial effects on asthma, the possibility of preventing and treating asthma cannot be denied. In addition, fecal microbiota transplantation (FMT) is another way to improve the gut microbiota, but its clinical application is currently limited in asthma. Additional studies are required to confirm the clinical stability and safety of both probiotic supplementation and FMT. In summary, the gut microbiota is closely associated with asthma, and its imbalance is related to an increased risk and severity of asthma, suggesting that appropriate gut microbiota intervention may be a feasible way to prevent and treat asthma.

Probiotics are also used in patients with autoimmune diseases, such as RA, which have been shown to be associated with the gut microbiota [47]. An early study reported that Lactobacillus salivarius, Lactobacillus iners, and Lactobacillus ruminis were increased in the gut of untreated RA patients, suggesting that a relationship potentially exists between the Lactobacillus community and the development of RA [48]. RCTs have demonstrated beneficial the effects of Lactobacillus acidophilus, Lactobacillus casei, or Bifidobacterium bifidum in RA patients [49, 50]. In contrast, Pineda et al. found that Lactobacillus rhamnosus and Lactobacillus reuteri did not clinically improve RA [51]. Probiotics have been shown to regulate immune system function and affect inflammation in a strain-specific manner. Lactobacilli are probiotic bacteria; however, different Lactobacillus species 


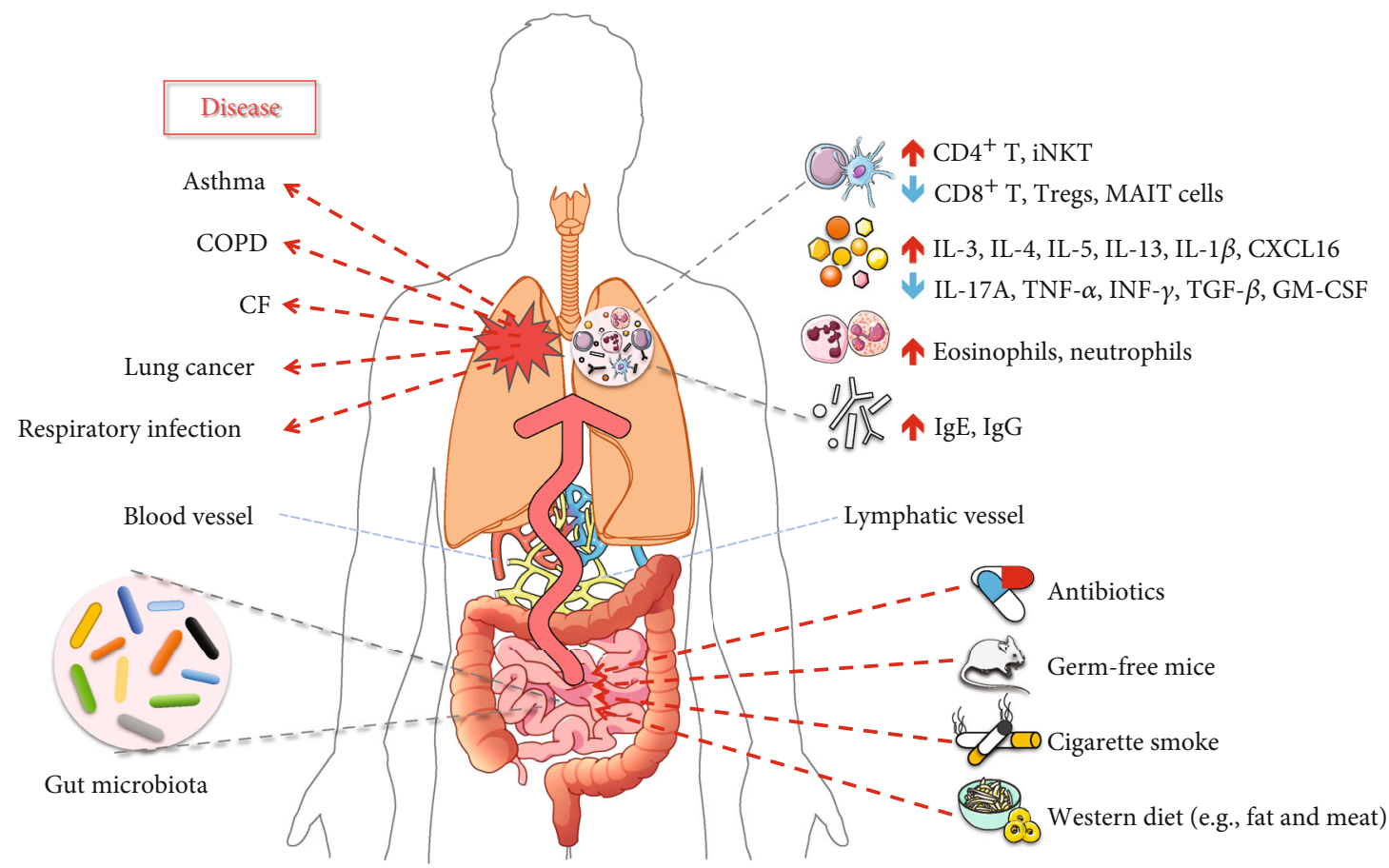

(a)

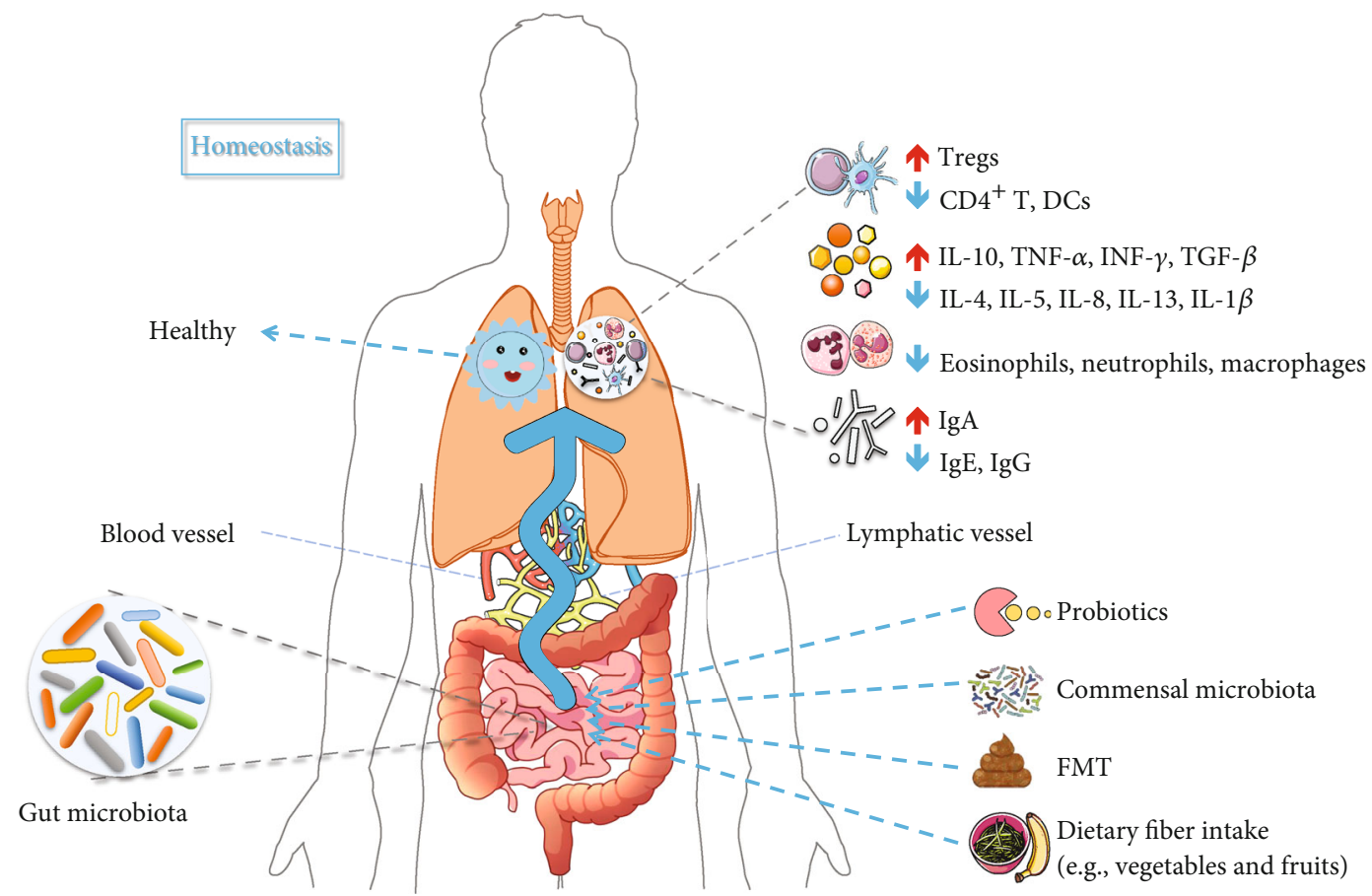

(b)

FIGURE 1: The role of gut microbiota in respiratory disease and homeostasis. The dysbiosis of gut microbiota contributes to respiratory diseases (a), while a healthy gut microbiota plays a protective role in the lung (b). The gut microbiota is influenced by several factors, including antibiotics, probiotics, cigarette smoke, diets, and fecal microbiota transplantation (FMT), and is associated with lung health and disease by regulating the respiratory immunity and inflammation through the blood and lymphatic system. $\uparrow:$ increase; $\downarrow$ : decrease.

have different effects on RA, and some Lactobacillus species may cause arthritis [52]. For example, Lactobacillus casei plays an important role in inducing arthritis, while Lactobacillus fermentum does not induce arthritis [53]. Therefore, regarding probiotic supplementation, it is highly important to choose a bacterial strain and dosage that are safe and beneficial.

3.2. COPD. COPD, a common chronic, preventable, and treatable respiratory disease, is characterized by persistent 
airflow limitation and increased airway inflammation. Worldwide, COPD has been a major public health problem because of its high prevalence, morbidity, and mortality. Although much evidence has shown a coexistence of COPD and chronic gastrointestinal diseases such as IBD, few studies have reported the gut microbiota in COPD patients. Smoking is the principal cause of developing COPD and is associated with the microbial community and immune response of the GI tract [54]. The gut microbiota changes along with different cigarette smoking statuses [55]; Biedermann et al. found an altered gut microbiota in healthy smokers compared with that in nonsmokers, and they further observed an increase in Actinobacteria and Firmicutes and a decrease in Bacteroidetes and Proteobacteria abundances after smoking cessation [56]. In a mouse study, Lachnospiraceae sp. was increased in the gut after smoke exposure [54]. Although few studies have identified the direct association between the gut microbiota and COPD, there is evidence that the gut microbiota may play a vital role in COPD induced by cigarette smoke.

Similarly, there have been relatively few studies about probiotics that revealed the connection between the gut microbiota and COPD. For example, intragastric supplementation with Lactobacillus rhamnosus and Bifidobacterium breve in mice with COPD attenuated airway inflammation and alveolar damage [57]. In vitro, these two probiotics showed a similar anti-inflammatory effect on cigarette smoke-induced inflammation in human macrophages [58]. In the future, additional studies conducted on COPD patients are required to investigate and confirm the role of probiotics in COPD and to provide new therapeutic strategies for COPD.

3.3. CF $\mathrm{CF}$, a common autosomal recessive disease that affects mainly the lungs, is primarily driven by cystic fibrosis transmembrane conductance regulator (CFTR) mutation. The GI tract also strongly presents CFTR dysfunction and is among the earliest parts of the body affected in CF patients, suggesting a close link between the gut and lung. In CF patients, the gut microbiota was significantly altered, with reduced bacterial abundance, richness, and diversity and different microbial compositions compared to those in HCs [59-61]. For example, increased abundances of Staphylococcus, Streptococcus, and Veillonella dispar and decreased abundances of Bacteroides, Bifidobacterium adolescentis, and Faecalibacterium prausnitzii were observed in the gut of CF patients compared with those of HCs [62]. Importantly, the gut microbiota, which was reported to be associated with CFTR variants [63], seems to be essential for the pathophysiology and development of CF. A murine study indicated that the loss of functional CFTR was associated with augmentation of pathogenic bacteria, such as Mycobacteria and Bacteroides fragilis [64]. Moreover, several crosssectional studies revealed a certain relationship between the gut microbiota and lung function, disease exacerbation, and severity of CF patients [65-67].

In recent years, numerous RCTs have shown that restoration of the gut microbiota followed by probiotic supplementation is related to improvement of CF, further strengthening the idea that the gut microbiota can influence airway inflam- mation in CF. Lactobacillus administration caused a reduction in bacterial density and an increase in microbial diversity in the gut [68], as well as beneficial effects on exacerbation risk and quality of life in CF patients [69]. However, some inconsistent results were also yielded; for example, Van Biervliet et al. found no significant differences in pulmonary function and disease exacerbations between probiotic and placebo groups [70]. Therefore, according to a meta-analysis, fastidiously designed and adequate RCTs are needed to assess the safety and efficacy of probiotics and to ascertain the specific probiotic strains or dose that can be of significant benefit for CF patients [71].

3.4. Lung Cancer. Lung cancer is one of the malignant tumors with the fastest growth of morbidity and mortality and has become the greatest threat to human health. Antibiotics are believed to alter the gut microbiota, and a large demographic study found that exposure to certain antibiotics, such as penicillin, cephalosporins, or macrolides, was associated with an increased risk of lung cancer [72], which suggested a close correlation between the gut microbiota and lung cancer. Using $16 \mathrm{~S}$ rRNA sequencing, researchers found no significant difference in alpha diversity but a difference in gut microbiota beta diversity between patients with lung cancer and HCs [73, 74]. Moreover, at the phylum and genus levels, lung cancer patients had an increased abundance of Enterococcus and a reduced level of the phylum Actinobacteria and genus Bifidobacterium, and these microbial communities might be potential biomarkers for lung carcinogenesis [73].

Recently, several studies have indicated that the gut microbiota also contributes to the effect of lung cancer therapeutics. Patients with non-small-cell lung cancer (NSCLC) who responded to antiprogrammed death 1 (PD-1) immunotherapy (responders) harbored a higher gut microbial diversity than those who did not respond (nonresponders), and gut microbial diversity was positively associated with progression-free survival (PFS) [75]. Another study revealed that responders showed increased abundances of Akkermansia muciniphila, Ruminococcus, Eubacterium, and Alistipes and decreased abundances of Bifidobacterium and Parabacteroides in the gut compared with those of nonresponders [76]. In addition, antibiotic use can influence the efficacy of lung cancer therapy. Previous studies found that antibiotics before and during antitumor therapy significantly reduced the clinical benefit (PFS and overall survival) of antitumor drugs in patients with NSCLC [77, 78]. Furthermore, FMT into GF or antibiotic-treated mice can ameliorate antitumor effects and clinical activity of antitumor drugs [79]. Similarly, supplementation with Enterococcus hirae and Barnesiella intestinihominis can prolong PFS in mice with advanced lung cancer undergoing chemoimmunotherapy [80]. Taken together, the gut microbiota markedly influences the outcome of antitumor therapy for lung cancer, suggesting a potential strategy to improve the clinical outcomes of patients with lung cancer by modulating the gut microbiota. However, a large number of clinical studies are required to confirm the effectiveness and safety of FMT and probiotics. 
3.5. Respiratory Infection. Respiratory infection is the most common infectious disease and is a leading cause of morbidity and mortality worldwide. The gut commensal microbiota provides essential benefits to pulmonary mucosal immunity and plays protective roles in respiratory infection by distally driving host responses to pneumonia [81]. Depletion or absence of the gut microbiota is believed to influence the host immune response. Schuijt et al. found that microbiota-depleted mice showed increased bacterial dissemination, inflammation, organ damage, and mortality compared with control mice, and FMT reversed the gut microbiota diversity and enhanced the host defense against pneumonia [82]. In addition, the gut microbiota differed between patients with respiratory infection and HCs. It was reported that certain gut microbiota, such as Enterococcaceae, was associated with community-acquired pneumonia (CAP) [83], and respiratory syncytial virus and influenza virus infection resulted in a dysbiotic gut microbiota in mice [84]. Many studies have suggested that oral administration of probiotics can not only protect against bacterial pneumonia [85] but also contribute to accelerated recovery from respiratory viral infection [86, 87], further emphasizing the crucial role of the gut microbiota in respiratory infection.

Tuberculosis (TB) typically affects the lungs (pulmonary $\mathrm{TB}$ ), causing approximately 10 million cases and over 1 million deaths per year worldwide, with the heaviest toll in lowand middle-income countries [88]. Likewise, the gut commensal microbiota can protect against early lung colonization by Mycobacterium tuberculosis (Mtb) [89]. Disruption of the gut microbiota with antibiotics increased the burden and dissemination of $M t b$, and FMT reconstituted the gut microbiota and restored TB containment by reducing the $M t b$ burden [90]. The gut microbiota was significantly different between patients with TB and HCs. At the phylum level, Actinobacteria and Proteobacteria, which contain many pathogenic species, were enriched in the gut of TB patients, while Bacteroidetes, which contains a variety of beneficial commensal microbiota species, was decreased in TB patients compared to those in HCs [91]. At the genus level, several butyrate and propionate-producing bacteria, such as Faecalibacterium, Roseburia, Eubacterium, and Phascolarctobacterium, were more abundant in TB patients than in HCs [92]. Similar to antibiotic and antitumor therapy, anti-TB treatment also has dramatic effects on the gut microbiota. Patients who underwent standard HRZE (isoniazid, rifampicin, pyrazinamide, and ethambutol) therapy exhibited a perturbed gut microbiota, with a depletion of Ruminococcus, Eubacterium, Lactobacillus, and Bacteroides and an increase in Erysipelatoclostridium and Prevotella abundances [93], and HRZ(E)induced dysbiosis was long lasting in both mice and humans [94]. Furthermore, studies on probiotics suggested that supplementation with Lactobacillus can restore anti-Mtb immunity in the lungs [95]. Taken together, these findings indicate that the gut microbiota may contribute to the pathophysiology of TB.

3.6. Other Respiratory Diseases. In addition to the above common respiratory diseases, other respiratory disorders, such as
ILD, acute respiratory distress syndrome (ARDS), acute lung injury (ALI), and ventilator-associated pneumonia (VAP), also show a certain correlation with the gut microbiota. For example, ILD is characterized by progressive fibrosis and respiratory failure, and changes in the gut microbiota have been reported in patients with silicosis and pulmonary fibrosis [96]. ARDS/ALI is the most common form of organ failure in critically ill patients, and VAP, which is among the most common infections in mechanically ventilated patients, has a high mortality rate $[97,98]$. Previous studies revealed that gut-associated bacteria and pathogens were enriched in the bronchoalveolar lavage fluid (BALF) of patients with ARDS, which suggested gut-lung translocation [99, 100]. Additionally, the GI microbiota contributes to the development of ALI in mice [101], and FMT can significantly reduce ALI inflammation [102]. RCTs of ventilated patients suggested that patients treated with probiotics had a decreased incidence of microbiologically confirmed VAP, as well as reduced durations of intensive care unit [103] and hospital stays [104]. A meta-analysis also found an association between probiotic supplementation and reduced VAP incidence, suggesting a clinical benefit of probiotics for ventilated patients [105].

\section{The Complex Interactions between the Gut and Lungs}

Cigarette smoking, which is a risk factor for many diseases, can not only change the lung microbiota but also affect the gut microbiota $[55,106]$. Lung mucosal exposure to cigarette smoke may be involved in the development of autoantibodies associated with RA, such as peptidylarginine deiminase (PAD) 2 [107], suggesting that the lungs could be a site of autoimmunity generation in RA. Scher et al. found that the lung microbiota in RA patients was similar to that in sarcoidosis patients, characterized by reduced alpha diversity and decreased abundance of Actinomyces and Burkholderia [108]. The notion that the gut microbiota influences the local and systemic immune systems is not novel, and the role of the gut microbiota in autoimmune diseases, including RA, is currently well characterized [47]. Since the lungs and gut are both mucosal sites that are exposed to environmental factors, it is possible that both organs share microbiota and that the microbiota can induce local and systemic immunity/inflammation in both organs. This evidence suggests that the gut/lung microbiota may potentially drive the initiation of autoimmune diseases.

Previous studies have found an altered function and structure in intestinal mucosa in asthma patients and increased intestinal permeability in COPD patients [109, 110], further supporting the hypothesis that a link exists between the gut and lungs. Moreover, a growing number of studies have suggested that an immunological relationship exists between the gut and lungs $[111,112]$. The gut microbiota can shape local intestinal and systemic immunity and the lung mucosa, thereby affecting respiratory diseases. Taken together, the complex interaction between the gut and lungs is likely to be mediated by locally resident microbiota. 
TABLe 1: The possible mechanisms underlying the effects of the gut microbiota on respiratory diseases.

\begin{tabular}{|c|c|c|c|}
\hline $\begin{array}{l}\text { Respiratory } \\
\text { diseases }\end{array}$ & $\begin{array}{l}\text { Alterations in the } \\
\text { gut microbiota }\end{array}$ & Possible mechanisms & References \\
\hline \multirow{9}{*}{ Asthma } & \multirow{3}{*}{$\begin{array}{l}\text { Gut microbiota } \\
\text { disrupted by } \\
\text { antibiotics }\end{array}$} & $\begin{array}{l}\text { Exacerbate Th2 responses by increasing the infiltration of inflammatory } \\
\text { cells and the production of inflammatory cytokines (IL- } 4 \text { and IL-13). }\end{array}$ & {$[117,118]$} \\
\hline & & Reduce Treg abundance in the lung. & [119] \\
\hline & & Exaggerate Th1/Th17 adaptive immune responses in the lung. & {$[120]$} \\
\hline & \multirow[t]{2}{*}{ GF mice } & $\begin{array}{l}\text { Elevate the total number of eosinophils, number of } \mathrm{CD}^{+} \mathrm{T} \text { cells, } \\
\text { and level of Th2 cytokines and alter the number and phenotype of } \\
\text { conventional DCs in the airways. }\end{array}$ & {$[114]$} \\
\hline & & Increase CXCL16 expression and accumulate iNKT cells in the gut and lungs. & {$[121]$} \\
\hline & \multirow{4}{*}{ Probiotics } & $\begin{array}{l}\text { Reverse the Th1/Th2 imbalance: increase the levels of the anti-inflammatory } \\
\text { cytokine IL-10 while reduce the levels of proinflammatory cytokines such as } \\
\text { IL- } 4 \text {, IL-5, and IL- } 13 \text {. }\end{array}$ & {$[122-125]$} \\
\hline & & Increase PPAR $\gamma$ expression of DCs in the lung. & [126] \\
\hline & & $\begin{array}{l}\text { Increase lung } \mathrm{CD}^{+} \mathrm{T} \text { cell and } \mathrm{CD} 4^{+} \mathrm{Foxp}^{+} \text {Treg abundance while decrease } \\
\text { activated } \mathrm{CD} 11 \mathrm{~b}^{+} \mathrm{DC} \text { abundance. }\end{array}$ & [37] \\
\hline & & $\begin{array}{l}\text { Decrease MMP9 expression in the BALF and serum and inhibit inflammatory } \\
\text { cell infiltration into the lung. }\end{array}$ & {$[36]$} \\
\hline \multirow{4}{*}{ COPD } & \multirow[t]{2}{*}{ Cigarette smoke } & $\begin{array}{l}\text { Alter mucin gene expression and cytokine production in the gut; increase } \\
\text { Muc2, Muc3, and Muc4 expression; and increase CXCL2 and IL-6 expression } \\
\text { while decrease IFN- } \gamma \text { and TGF- } \beta \text { expression. }\end{array}$ & [54] \\
\hline & & $\begin{array}{l}\text { Inhibit the NK- } \kappa \mathrm{B} \text { pathway by reducing p } 65 \text { phosphorylation and } \\
\qquad \mathrm{I} \kappa \mathrm{B} \alpha \text { in the gut. }\end{array}$ & [127] \\
\hline & \multirow[t]{2}{*}{ Probiotics } & $\begin{array}{l}\text { Suppress macrophage inflammation by inducing the expression of IL- } 1 \beta \text {, } \\
\text { IL-6, IL-10, IL-23, TNF- } \alpha \text {, CXCL- } 8 \text {, and HMGB1. }\end{array}$ & [58] \\
\hline & & Increase NK cell activity and the number of $\mathrm{CD} 16^{+}$cells. & {$[128]$} \\
\hline \multirow{3}{*}{$\mathrm{CF}$} & \multirow{2}{*}{ Probiotics } & Reduce IL- 8 production by intestinal cells. & {$[129]$} \\
\hline & & Reduce the level of the gut inflammatory marker calprotectin. & {$[68]$} \\
\hline & Antibiotic treatment & $\begin{array}{l}\text { Augment the proportions of Th17, } \mathrm{CD}^{+} \mathrm{IL}^{-17^{+}} \text {, and } \mathrm{CD} 8^{+} \mathrm{IFN} \gamma^{+} \\
\text {lymphocytes and IL-17-producing } \gamma \delta \mathrm{T} \text { cells. }\end{array}$ & {$[130]$} \\
\hline \multirow{5}{*}{ Lung cancer } & \multirow{2}{*}{$\begin{array}{l}\text { Gut microbiota } \\
\text { disrupted by } \\
\text { antibiotics }\end{array}$} & $\begin{array}{l}\text { Upregulate the expression of VEGFA and downregulate the expression } \\
\text { of BAX and CDKN1B while reduce IFN- } \gamma \text {, GZMB, and PRF1 produced } \\
\text { by CD8 }{ }^{+} \text {T cells. }\end{array}$ & {$[131]$} \\
\hline & & $\begin{array}{l}\text { Suppress CTX-induced Th17 responses and reduce the abundance } \\
\text { of tumor-infiltrating } \mathrm{CD}^{+} \mathrm{T} \text { cells and Th1 cells. }\end{array}$ & {$[132]$} \\
\hline & FMT & Accumulate CCR $9^{+} \mathrm{CXCR}^{+} \mathrm{CD}^{+}{ }^{+} \mathrm{T}$ cells into the tumor microenvironment. & [79] \\
\hline & \multirow[b]{2}{*}{ Probiotics } & Upregulate the mRNA levels of IFN- $\gamma$, GZMB, and PRF1. & [131] \\
\hline & & $\begin{array}{l}\text { Boost CTX-induced anticancer Th1 and Tc1 responses and promote the } \\
\text { infiltration of IFN- } \gamma^{+} \gamma \delta \text { T cells into cancer lesions. }\end{array}$ & [80] \\
\hline \multirow{9}{*}{$\begin{array}{l}\text { Respiratory } \\
\text { infection }\end{array}$} & \multirow{5}{*}{$\begin{array}{l}\text { Commensal gut } \\
\text { microbiota }\end{array}$} & $\begin{array}{l}\text { SFB promotes pulmonary Th17 immunity as demonstrated by increased } \\
\text { IL-22 and IL- } 22^{+} \text {TCR } \beta^{+} \text {cell levels. }\end{array}$ & [133] \\
\hline & & $\begin{array}{c}\text { Protect against } M t b \text { infection by improving the activity of MAIT } \\
\text { cells in the lungs. }\end{array}$ & [89] \\
\hline & & Regulate virus-specific CD4 and CD8 T cell and antibody responses. & [134] \\
\hline & & Contribute to the accumulation of IL-22-producing ILC3s in newborn lung. & {$[81]$} \\
\hline & & Induce NF- $\kappa \mathrm{B}$ activation in the lung through TLR4. & {$[135,136]$} \\
\hline & \multirow{4}{*}{$\begin{array}{l}\text { Gut microbiota } \\
\text { disrupted by } \\
\text { antibiotics }\end{array}$} & Reduce pulmonary GM-CSF production through IL-17A signaling. & [115] \\
\hline & & Reduce MAIT cell and IL-17A levels. & [89] \\
\hline & & Reduce mincle expression on lung DCs. & [95] \\
\hline & & $\begin{array}{l}\text { Decrease bacterial killing activity of alveolar macrophages while increase } \\
\text { the levels of proinflammatory cytokines such as IL- } 6 \text { and IL- } 1 \beta \text { in the lung. }\end{array}$ & {$[136]$} \\
\hline
\end{tabular}


TABLe 1: Continued.

\begin{tabular}{|c|c|c|c|}
\hline $\begin{array}{l}\text { Respiratory } \\
\text { diseases }\end{array}$ & $\begin{array}{l}\text { Alterations in the } \\
\text { gut microbiota }\end{array}$ & Possible mechanisms & References \\
\hline & GF mice & $\begin{array}{l}\text { Decrease proinflammatory cytokine (TNF- } \alpha \text { and CXCL1) levels and } \\
\text { neutrophil influx while produce large amounts of IL-10 in the lungs. }\end{array}$ & [137] \\
\hline & FMT & Normalize the pulmonary TNF- $\alpha$ and IL-10 levels. & {$[82]$} \\
\hline & & Activate the TLR-signaling pathway through the protein Mal. & {$[85]$} \\
\hline & Probiotics & $\begin{array}{l}\text { Enhance the mRNA expression of IFN- } \gamma \text {, IL-12a, IL-2rb, IL-12rb1, PRF1, } \\
\text { Klrk1, CD247, and TNF- } \alpha \text { in the lung. }\end{array}$ & {$[138]$} \\
\hline ALI & FMT & $\begin{array}{l}\text { Reduce TNF- } \alpha \text {, IL- } 1 \beta \text {, and IL-6 levels by downregulating the } \\
\text { TGF- } \beta 1 / \text { Smads/ERK signaling pathway. }\end{array}$ & {$[102]$} \\
\hline \multicolumn{4}{|c|}{$\begin{array}{l}\text { ALI: acute lung injury; BAX: Bcl-2 associated X; CDKN1B: cyclin-dependent kinase inhibitor 1B; CTX: cyclophosphamide; CXCL: C-X-C motif chemokine } \\
\text { ligand; DCs: dendritic cells; ERK: extracellular signal-regulated kinase; FMT: fecal microbiota transplantation; GF mice: germ-free mice; GM-CSF: } \\
\text { granulocyte-macrophage colony-stimulating factor; GZMB: granzyme B; HMGB1: high-mobility group box 1; IFN- } \gamma \text { : interferon-gamma; I } \kappa \text { B } \alpha \text { : inhibitor of } \\
\text { NK- } \kappa \mathrm{B} \alpha \text {; Klrk1: killer cell lectin-like receptor subfamily K, member 1; MAIT cells: mucosal-associated invariant T cells; Mal: MyD88 (myeloid } \\
\text { differentiation primary response protein) adaptor protein; MMP9: matrix metalloproteinase 9; Muc: mucin; NF- } \kappa \mathrm{B} \text { : nuclear factor kappa-B; PPAR } \gamma \text { : } \\
\text { peroxisome proliferator-activated receptor gamma; PRF1: perforin; SFB: segmented filamentous bacteria; TLR: toll-like receptor; TNF- } \alpha \text { : tumor necrosis } \\
\text { factor alpha; Tregs: regulatory T cells; VEGFA: vascular endothelial growth factor A. }\end{array}$} \\
\hline
\end{tabular}

\section{Possible Mechanisms of the Gut Microbiota in Respiratory Diseases}

The gut commensal microbiota contributes to influencing and maintaining body homeostasis by regulating the immune response of both the GI system and distal organs. The possible mechanisms include the regulation of extraintestinal $\mathrm{T}$ cell populations, development of oral immune tolerance through regulatory $\mathrm{T}$ cells (Tregs), production of short-chain fatty acids (SCFAs), and regulation of systemic inflammation [113]. The immune cells and cytokines induced by the gut microbiota and its metabolites, such as SCFAs, can enter systemic circulation through the blood and lymphatic system, which regulate the immune and inflammatory responses in the lung and further influence respiratory health and disease (Figure 1). For example, exaggerated allergic airway inflammation in GF mice was correlated with increased T helper 2 (Th2) cytokine (IL-4 and IL-5) and IgE levels in the lung [114]. The commensal gut microbiota can enhance host defense against bacterial pneumonia by increasing IL-17A levels and upregulating pulmonary granulocyte-macrophage colony-stimulating factor (GM-CSF) signaling [115]. In antibiotic-treated mice, a greatly increased mortality due to respiratory viral infection was related to a decreased abundance of Tregs in the respiratory and GI tracts [116], and increased pulmonary colonization by $M t b$ was associated with a significantly reduced accumulation of mucosal-associated invariant T (MAIT) cells in the lungs [89]. Table 1 summarizes our current understanding of the possible mechanisms of the gut microbiota acting on common respiratory diseases.

Currently, the mechanisms of probiotic regulation of lung health and disease have become a research hotspot since there is increasing evidence that probiotics have protective and therapeutic effects on respiratory diseases by optimizing microbial balance in the GI tract (Table 1). Oral administration of probiotics contributes to regulating respiratory immune responses through numerous signaling pathways. For example, Bifidobacterium bifidum can stimulate the Th1/Th2 balance and upregulate IFN- $\gamma$, IL-4, and IL-
12 secretion in the spleen [139]; Escherichia coli can reduce respiratory inflammatory cell recruitment as well as Th2 and Th17 responses [140]; Enterococcus faecalis suppresses Th17 cell development in the lung, spleen, and gut [141]; and Lactobacillus plantarum can reduce the numbers of lung innate immune cells (macrophages and neutrophils) and levels of cytokines (IL- 6 and TNF- $\alpha$ ) in the BALF and induce an immunosuppressive Treg response in the lungs [142]. Despite these effects, the precise mechanisms underlying probiotic effects on the lung and many aspects of the probiotic regulation of immune responses remain largely unknown.

\section{Conclusions}

Increasing evidence suggests an important and complex crosstalk between the gut and lung, as well as between the gut microbiota and host immunity. Gut microbial dysbiosis is believed to be associated with the etiology or/and development of common respiratory diseases, such as asthma, COPD, CF, lung cancer, and respiratory infection. To date, the understanding of the mechanism involving the gut-lung axis is still in its infancy and remains to be further elucidated. Future research into modification and improvement of the gut microbiota and into the balance of gut and lung immunity through diet, probiotics, and FMT is necessary to improve our understanding of the role of gut microbiota in the lung and to provide effective and new therapeutic strategies for respiratory diseases.

\section{Conflicts of Interest}

The authors declare no conflict of interests.

\section{Acknowledgments}

We thank American Journal Experts (AJE) for English language editing. This work was supported by the Guangzhou Healthcare Collaborative Innovation Major Project 
(201604020012), the Science and Technology Planning Project of Guangdong Province (2016A020215107), the Open Project of the State Key Laboratory of Respiratory Disease (SKLRD2016OP014), and the Open Projects Program of Guangdong Provincial Key Laboratory of Occupational Disease Prevention and Treatment (2017B030314152).

\section{References}

[1] G. Labarca, L. Drake, G. Horta et al., "Association between inflammatory bowel disease and chronic obstructive pulmonary disease: a systematic review and meta-analysis," $B M C$ Pulmonary Medicine, vol. 19, no. 1, pp. 186-186, 2019.

[2] M. Vutcovici, P. Brassard, and A. Bitton, "Inflammatory bowel disease and airway diseases," World Journal of Gastroenterology, vol. 22, no. 34, pp. 7735-7741, 2016.

[3] J. Wang, F. Li, H. Wei, Z. X. Lian, R. Sun, and Z. Tian, "Respiratory influenza virus infection induces intestinal immune injury via microbiota-mediated Th17 cell-dependent inflammation," Journal of Experimental Medicine, vol. 211, no. 12, pp. 2397-2410, 2014.

[4] A. Massart and D. P. Hunt, "Pulmonary manifestations of inflammatory bowel disease," The American Journal of Medicine, vol. 133, no. 1, pp. 39-43, 2020.

[5] A. J. Blanco, A. G. Vélez, G. Solís-García, A. S. Posadas, S. B. Alonso, and J. L. R. Cimadevilla, "Comorbidities and course of lung function in patients with congenital esophageal atresia," Archivos Argentinos de Pediatría, vol. 118, no. 1, pp. 25-30, 2020.

[6] Q. Feng, W.-D. Chen, and Y.-D. Wang, "Gut microbiota: an integral moderator in health and disease," Frontiers in Microbiology, vol. 9, p. 151, 2018.

[7] P. Gholizadeh, M. Mahallei, A. Pormohammad et al., "Microbial balance in the intestinal microbiota and its association with diabetes, obesity and allergic disease," Microbial Pathogenesis, vol. 127, pp. 48-55, 2019.

[8] K. F. Budden, S. L. Gellatly, D. L. A. Wood et al., "Emerging pathogenic links between microbiota and the gut-lung axis," Nature Reviews. Microbiology, vol. 15, no. 1, pp. 55-63, 2017.

[9] H. S. Hayden, A. Eng, C. E. Pope et al., "Fecal dysbiosis in infants with cystic fibrosis is associated with early linear growth failure," Nature Medicine, vol. 26, no. 2, pp. 215221, 2020.

[10] R. Bingula, M. Filaire, N. Radosevic-Robin et al., "Desired turbulence? Gut-lung axis, immunity, and lung cancer," Journal of Oncology, vol. 2017, Article ID 5035371, 15 pages, 2017.

[11] A. Dumas, L. Bernard, Y. Poquet, G. Lugo-Villarino, and O. Neyrolles, "The role of the lung microbiota and the gutlung axis in respiratory infectious diseases," Cellular Microbiology, vol. 20, no. 12, article e12966, 2018.

[12] P. Gerard, "Gut microbiota and obesity," Cellular and Molecular Life Sciences, vol. 73, no. 1, pp. 147-162, 2016.

[13] S. Kim, A. Covington, and E. G. Pamer, "The intestinal microbiota: antibiotics, colonization resistance, and enteric pathogens," Immunological Reviews, vol. 279, no. 1, pp. 90$105,2017$.

[14] K. P. Lemon, V. Klepac-Ceraj, H. K. Schiffer, E. L. Brodie, S. V. Lynch, and R. Kolter, "Comparative analyses of the bacterial microbiota of the human nostril and oropharynx," MBio, vol. 1, no. 3, p. e00129, 2010.
[15] R. P. Dickson, J. R. Erb-Downward, F. J. Martinez, and G. B. Huffnagle, "The microbiome and the respiratory tract," Annual Review of Physiology, vol. 78, no. 1, pp. 481-504, 2016.

[16] V. Robles Alonso and F. Guarner, "Intestinal microbiota composition in adults," in Probiotic Bacteria and Their Effect on Human Health and Well-Being, vol. 107, pp. 17-24, Karger Publishers, 2013.

[17] F. Frati, C. Salvatori, C. Incorvaia et al., "The role of the microbiome in asthma: the gut(-)lung axis," International Journal of Molecular Sciences, vol. 20, no. 1, p. E123, 2019.

[18] B. J. Marsland, A. Trompette, and E. S. Gollwitzer, "The gutlung axis in respiratory disease," Annals of the American Thoracic Society, vol. 12, Supplement 2, pp. S150-S156, 2015.

[19] E. Mitre, A. Susi, L. E. Kropp, D. J. Schwartz, G. H. Gorman, and C. M. Nylund, "Association between use of acidsuppressive medications and antibiotics during infancy and allergic diseases in early childhood," JAMA Pediatrics, vol. 172, no. 6, article e180315, 2018.

[20] J. Ni, H. Friedman, B. C. Boyd et al., "Early antibiotic exposure and development of asthma and allergic rhinitis in childhood," BMC Pediatrics, vol. 19, no. 1, p. 225, 2019.

[21] A. M. Cepeda, the ISAAC Phase III Latin America Group, S. Thawer et al., "Diet and respiratory health in children from 11 Latin American countries: evidence from ISAAC phase III," Lung, vol. 195, no. 6, pp. 683-692, 2017.

[22] F. Rusconi, D. Zugna, I. Annesi-Maesano et al., "Mode of delivery and asthma at school age in 9 European birth cohorts," American Journal of Epidemiology, vol. 185, no. 6, pp. 465-473, 2017.

[23] F. Ahmadizar, S. J. H. Vijverberg, H. G. M. Arets et al., "Breastfeeding is associated with a decreased risk of childhood asthma exacerbations later in life," Pediatric Allergy and Immunology, vol. 28, no. 7, pp. 649-654, 2017.

[24] M. L. Dreher, "Whole fruits and fruit fiber emerging health effects," Nutrients, vol. 10, no. 12, p. 1833, 2018.

[25] K. Loverdos, G. Bellos, L. Kokolatou et al., "Lung microbiome in asthma: current perspectives," Journal of Clinical Medicine, vol. 8, no. 11, p. 1967, 2019.

[26] X. Zhang, D. Zhang, H. Jia et al., "The oral and gut microbiomes are perturbed in rheumatoid arthritis and partly normalized after treatment," Nature Medicine, vol. 21, no. 8, pp. 895-905, 2015.

[27] A. P. Diamanti, M. M. Rosado, B. Laganà, and R. D'Amelio, "Microbiota and chronic inflammatory arthritis: an interwoven link," Journal of Translational Medicine, vol. 14, no. 1, pp. 233-233, 2016.

[28] D. Daley, "The evolution of the hygiene hypothesis: the role of early-life exposures to viruses and microbes and their relationship to asthma and allergic diseases," Current Opinion in Allergy and Clinical Immunology, vol. 14, no. 5, pp. 390-396, 2014.

[29] T. R. Abrahamsson, H. E. Jakobsson, A. F. Andersson, B. Björkstén, L. Engstrand, and M. C. Jenmalm, "Low gut microbiota diversity in early infancy precedes asthma at school age," Clinical and Experimental Allergy, vol. 44, no. 6, pp. 842850, 2014.

[30] W. H. Oddy, "Breastfeeding, childhood asthma, and allergic disease," Annals of Nutrition and Metabolism, vol. 70, no. 2, pp. 26-36, 2017. 
[31] Q. Wang, F. Li, B. Liang et al., "A metagenome-wide association study of gut microbiota in asthma in UK adults," BMC Microbiology, vol. 18, no. 1, p. 114, 2018.

[32] K. E. Fujimura, A. R. Sitarik, S. Havstad et al., "Neonatal gut microbiota associates with childhood multisensitized atopy and T cell differentiation," Nature Medicine, vol. 22, no. 10, pp. 1187-1191, 2016.

[33] F. A. van Nimwegen, J. Penders, E. E. Stobberingh et al., "Mode and place of delivery, gastrointestinal microbiota, and their influence on asthma and atopy," Journal of Allergy and Clinical Immunology, vol. 128, no. 5, pp. 948-955.e3, 2011.

[34] M.-C. Arrieta, L. T. Stiemsma, P. A. Dimitriu et al., "Early infancy microbial and metabolic alterations affect risk of childhood asthma," Science Translational Medicine, vol. 7, no. 307, article 307ra152, 2015.

[35] H. Kozakova, M. Schwarzer, L. Tuckova et al., "Colonization of germ-free mice with a mixture of three lactobacillus strains enhances the integrity of gut mucosa and ameliorates allergic sensitization," Cellular \& Molecular Immunology, vol. 13, no. 2, pp. 251-262, 2016.

[36] C. T. Wu, P. J. Chen, Y. T. Lee, J. L. Ko, and K. H. Lue, "Effects of immunomodulatory supplementation with Lactobacillus rhamnosus on airway inflammation in a mouse asthma model," Journal of Microbiology, Immunology, and Infection. Wei Mian Yu Gan Ran Za Zhi, vol. 49, no. 5, pp. 625-635, 2016.

[37] E. J. Raftis, M. I. Delday, P. Cowie et al., "Bifidobacterium breve MRx0004 protects against airway inflammation in a severe asthma model by suppressing both neutrophil and eosinophil lung infiltration," Scientific Reports, vol. 8, no. 1, p. 12024, 2018.

[38] S. Kallio, A. K. Kukkonen, E. Savilahti, and M. Kuitunen, "Perinatal probiotic intervention prevented allergic disease in a caesarean-delivered subgroup at 13-year follow-up," Clinical and Experimental Allergy, vol. 49, no. 4, pp. 506515, 2019.

[39] C.-F. Huang, W.-C. Chie, and I.-J. Wang, "Efficacy of Lactobacillus administration in school-age children with asthma: a randomized, placebo-controlled trial," Nutrients, vol. 10, no. 11, article e1678, p. 1678, 2018.

[40] J. Lin, Y. Zhang, C. He, and J. Dai, "Probiotics supplementation in children with asthma: a systematic review and metaanalysis," Journal of Paediatrics and Child Health, vol. 54, no. 9, pp. 953-961, 2018.

[41] G. Davies, S. Jordan, C. J. Brooks et al., "Long term extension of a randomised controlled trial of probiotics using electronic health records," Scientific Reports, vol. 8, no. 1, article 7668, 2018.

[42] R. M. Schmidt, R. Pilmann Laursen, S. Bruun et al., "Probiotics in late infancy reduce the incidence of eczema: a randomized controlled trial," Pediatric Allergy and Immunology, vol. 30, no. 3, pp. 335-340, 2019.

[43] M. A. Rose, F. Stieglitz, A. Köksal, R. Schubert, J. Schulze, and S. Zielen, "Efficacy of probiotic Lactobacillus GG on allergic sensitization and asthma in infants at risk," Clinical and Experimental Allergy, vol. 40, no. 9, pp. 1398-1405, 2010.

[44] K. Stockert, B. Schneider, G. Porenta, R. Rath, H. Nissel, and I. Eichler, "Laser acupuncture and probiotics in school age children with asthma: a randomized, placebo-controlled pilot study of therapy guided by principles of traditional Chinese medicine," Pediatric Allergy and Immunology, vol. 18, no. 2, pp. 160-166, 2007.

[45] A. K. Kukkonen, M. Kuitunen, E. Savilahti, A. Pelkonen, P. Malmberg, and M. Mäkelä, "Airway inflammation in probiotic-treated children at 5 years," Pediatric Allergy and Immunology, vol. 22, no. 2, pp. 249-251, 2011.

[46] T. D. H. Smith, H. Watt, L. Gunn, J. Car, and R. J. Boyle, "Recommending oral probiotics to reduce winter antibiotic prescriptions in people with asthma: a pragmatic randomized controlled trial," Annals of Family Medicine, vol. 14, no. 5, pp. 422-430, 2016.

[47] J. C. Clemente, J. Manasson, and J. U. Scher, "The role of the gut microbiome in systemic inflammatory disease," $B M J$, vol. 360, p. j5145, 2018.

[48] X. Liu, Q. Zou, B. Zeng, Y. Fang, and H. Wei, “Analysis of fecal Lactobacillus community structure in patients with early rheumatoid arthritis," Current Microbiology, vol. 67, no. 2, pp. 170-176, 2013.

[49] B. Zamani, H. R. Golkar, S. Farshbaf et al., "Clinical and metabolic response to probiotic supplementation in patients with rheumatoid arthritis: a randomized, double-blind, placebocontrolled trial," International Journal of Rheumatic Diseases, vol. 19, no. 9, pp. 869-879, 2016.

[50] B. Alipour, A. Homayouni-Rad, E. Vaghef-Mehrabany et al., "Effects of Lactobacillus casei supplementation on disease activity and inflammatory cytokines in rheumatoid arthritis patients: a randomized double-blind clinical trial," International Journal of Rheumatic Diseases, vol. 17, no. 5, pp. 519527, 2014.

[51] M. de los Angeles Pineda, S. F. Thompson, K. Summers, F. de Leon, J. Pope, and G. Reid, "A randomized, double-blinded, placebo-controlled pilot study of probiotics in active rheumatoid arthritis," Medical Science Monitor, vol. 17, no. 6, pp. Cr347-Cr354, 2011.

[52] E. Simelyte, M. Rimpiläinen, X. Zhang, and P. Toivanen, "Role of peptidoglycan subtypes in the pathogenesis of bacterial cell wall arthritis," Annals of the Rheumatic Diseases, vol. 62, no. 10, pp. 976-982, 2003.

[53] E. Simelyte, M. Rimpiläinen, L. Lehtonen, X. Zhang, and P. Toivanen, "Bacterial cell wall-induced arthritis: chemical composition and tissue distribution of four Lactobacillus strains," Infection and Immunity, vol. 68, no. 6, pp. 35353540, 2000.

[54] L. Allais, F. M. Kerckhof, S. Verschuere et al., "Chronic cigarette smoke exposure induces microbial and inflammatory shifts and mucin changes in the murine gut," Environmental Microbiology, vol. 18, no. 5, pp. 1352-1363, 2016.

[55] S. H. Lee, Y. Yun, S. J. Kim et al., "Association between cigarette smoking status and composition of gut microbiota: population-based cross-sectional study," Journal of Clinical Medicine, vol. 7, no. 9, p. 282, 2018.

[56] L. Biedermann, J. Zeitz, J. Mwinyi et al., "Smoking cessation induces profound changes in the composition of the intestinal microbiota in humans," PloS One, vol. 8, no. 3, article e59260, 2013.

[57] K. A. T. Verheijden, J. van Bergenhenegouwen, J. Garssen, G. F. G. Bezemer, A. D. Kraneveld, and G. Folkerts, "Treatment with specific prebiotics or probiotics prevents the development of lung emphysema in a mouse model of COPD," European Journal of Pharmacology, vol. 668, pp. e12-e13, 2011. 
[58] E. Mortaz, I. M. Adcock, F. L. M. Ricciardolo et al., "Antiinflammatory effects of Lactobacillus rahmnosus and Bifidobacterium breve on cigarette smoke activated human macrophages," PloS One, vol. 10, no. 8, article e0136455, 2015.

[59] L. Li and S. Somerset, "The clinical significance of the gut microbiota in cystic fibrosis and the potential for dietary therapies," Clinical Nutrition, vol. 33, no. 4, pp. 571-580, 2014.

[60] G. Dayama, S. Priya, D. E. Niccum, A. Khoruts, and R. Blekhman, "Interactions between the gut microbiome and host gene regulation in cystic fibrosis," Genome Medicine, vol. 12, no. 1, p. 12, 2020.

[61] Y. Wang, L. E. X. Leong, R. L. Keating et al., "Opportunistic bacteria confer the ability to ferment prebiotic starch in the adult cystic fibrosis gut," Gut Microbes, vol. 10, no. 3, pp. 367-381, 2018.

[62] R. Enaud, K. B. Hooks, A. Barre et al., "Intestinal inflammation in children with cystic fibrosis is associated with Crohn's-like microbiota disturbances," Journal of Clinical Medicine, vol. 8, no. 5, p. 645, 2019.

[63] P. Vernocchi, F. Del Chierico, A. Russo et al., "Gut microbiota signatures in cystic fibrosis: loss of host CFTR function drives the microbiota enterophenotype," PloS One, vol. 13, no. 12, article e0208171, 2018.

[64] S. V. Lynch, K. C. Goldfarb, Y. K. Wild, W. Kong, R. C. De Lisle, and E. L. Brodie, "Cystic fibrosis transmembrane conductance regulator knockout mice exhibit aberrant gastrointestinal microbiota," Gut Microbes, vol. 4, no. 1, pp. 41-47, 2014.

[65] M. J. Coffey, S. Nielsen, B. Wemheuer et al., "Gut microbiota in children with cystic fibrosis: a taxonomic and functional dysbiosis," Scientific Reports, vol. 9, no. 1, p. 18593, 2019.

[66] A. G. Hoen, J. Li, L. A. Moulton et al., “Associations between gut microbial colonization in early life and respiratory outcomes in cystic fibrosis," The Journal of Pediatrics, vol. 167, no. 1, pp. 138-147.e3, 2015.

[67] D. G. Burke, F. Fouhy, M. J. Harrison et al., "The altered gut microbiota in adults with cystic fibrosis," BMC Microbiology, vol. 17 , no. 1 , p. $58,2017$.

[68] R. del Campo, M. Garriga, A. Pérez-Aragón et al., "Improvement of digestive health and reduction in proteobacterial populations in the gut microbiota of cystic fibrosis patients using a Lactobacillus reuteri probiotic preparation: A double blind prospective study," Journal of Cystic Fibrosis, vol. 13, no. 6, pp. 716-722, 2014.

[69] S. Van Biervliet, D. Declercq, and S. Somerset, "Clinical effects of probiotics in cystic fibrosis patients: a systematic review," Clinical Nutrition ESPEN, vol. 18, pp. 37-43, 2017.

[70] S. Van Biervliet, B. Hauser, S. Verhulst et al., "Probiotics in cystic fibrosis patients: a double blind crossover placebo controlled study: pilot study from the ESPGHAN Working Group on Pancreas/CF," Clinical Nutrition ESPEN, vol. 27, pp. 59-65, 2018.

[71] M. J. Coffey, M. Garg, N. Homaira, A. Jaffe, and C. Y. Ooi, "Probiotics for people with cystic fibrosis," Cochrane Database of Systematic Reviews, vol. 1, no. 1, 2020.

[72] B. Boursi, R. Mamtani, K. Haynes, and Y. X. Yang, "Recurrent antibiotic exposure may promote cancer formation-another step in understanding the role of the human microbiota?," European Journal of Cancer, vol. 51, no. 17, pp. 2655-2664, 2015.
[73] H. Zhuang, L. Cheng, Y. Wang et al., "Dysbiosis of the gut microbiome in lung cancer," Frontiers in Cellular and Infection Microbiology, vol. 9, p. 112, 2019.

[74] W. Q. Zhang, S. K. Zhao, J. W. Luo et al., "Alterations of fecal bacterial communities in patients with lung cancer," American Journal of Translational Research, vol. 10, no. 10, pp. 3171-3185, 2018.

[75] Y. Jin, H. Dong, L. Xia et al., "The diversity of gut microbiome is associated with favorable responses to anti-programmed death 1 immunotherapy in Chinese patients with NSCLC," Journal of Thoracic Oncology, vol. 14, no. 8, pp. 1378-1389, 2019.

[76] P. Biancheri, D. Divekar, and A. J. M. Watson, "Could fecal transplantation become part of PD-1-based immunotherapy, due to effects of the intestinal microbiome?," Gastroenterology, vol. 154, no. 6, pp. 1845-1847, 2018.

[77] T. Hakozaki, Y. Okuma, M. Omori, and Y. Hosomi, "Impact of prior antibiotic use on the efficacy of nivolumab for nonsmall cell lung cancer," Oncology Letters, vol. 17, no. 3, pp. 2946-2952, 2019.

[78] L. Derosa, M. D. Hellmann, M. Spaziano et al., "Negative association of antibiotics on clinical activity of immune checkpoint inhibitors in patients with advanced renal cell and non-small-cell lung cancer," Annals of Oncology, vol. 29, no. 6, pp. 1437-1444, 2018.

[79] B. Routy, E. Le Chatelier, L. Derosa et al., "Gut microbiome influences efficacy of PD-1-based immunotherapy against epithelial tumors," Science, vol. 359, no. 6371, pp. 91-97, 2018.

[80] R. Daillère, M. Vétizou, N. Waldschmitt et al., "Enterococcus hirae and Barnesiella intestinihominis Facilitate Cyclophosphamide-Induced Therapeutic Immunomodulatory Effects," Immunity, vol. 45, no. 4, pp. 931-943, 2016.

[81] J. Gray, K. Oehrle, G. Worthen, T. Alenghat, J. Whitsett, and H. Deshmukh, "Intestinal commensal bacteria mediate lung mucosal immunity and promote resistance of newborn mice to infection," Science Translational Medicine, vol. 9, no. 376, article eaaf9412, 2017.

[82] T. J. Schuijt, J. M. Lankelma, B. P. Scicluna et al., "The gut microbiota plays a protective role in the host defence against pneumococcal pneumonia," Gut, vol. 65, no. 4, pp. 575-583, 2016.

[83] X. Ren, Y. Gamallat, D. Liu et al., "The distribution characteristics of intestinal microbiota in children with communityacquired pneumonia under five years of age," Microbial Pathogenesis, vol. 142, article 104062, 2020.

[84] H. T. Groves, L. Cuthbertson, P. James, M. F. Moffatt, M. J. Cox, and J. S. Tregoning, "Respiratory disease following viral lung infection alters the murine gut microbiota," Frontiers in Immunology, vol. 9, p. 182, 2018.

[85] A. T. Vieira, V. M. Rocha, L. Tavares et al., "Control of Klebsiella pneumoniae pulmonary infection and immunomodulation by oral treatment with the commensal probiotic Bifidobacterium longum 51A," Microbes and Infection, vol. 18, no. 3, pp. 180-189, 2016.

[86] N. Waki, N. Yajima, H. Suganuma et al., "Oral administration of Lactobacillus brevis KB290 to mice alleviates clinical symptoms following influenza virus infection," Letters in Applied Microbiology, vol. 58, no. 1, pp. 87-93, 2014.

[87] T. Kawahara, T. Takahashi, K. Oishi et al., "Consecutive oral administration of Bifidobacterium longum $\mathrm{MM}-2$ 
improves the defense system against influenza virus infection by enhancing natural killer cell activity in a murine model," Microbiology and Immunology, vol. 59, no. 1, pp. 112, 2015.

[88] WHO, Global tuberculosis report 2019, World Health Organization, 2019.

[89] A. Dumas, D. Corral, A. Colom et al., "The host microbiota contributes to early protection against lung colonization by Mycobacterium tuberculosis," Frontiers in Immunology, vol. 9, p. 2656, 2018.

[90] N. Khan, A. Vidyarthi, S. Nadeem, S. Negi, G. Nair, and J. N. Agrewala, "Alteration in the gut microbiota provokes susceptibility to tuberculosis," Frontiers in Immunology, vol. 7, p. 529, 2016.

[91] M. Luo, Y. Liu, P. Wu et al., "Alternation of gut microbiota in patients with pulmonary tuberculosis," Frontiers in Physiology, vol. 8, p. 822, 2017.

[92] A. Maji, R. Misra, D. B. Dhakan et al., "Gut microbiome contributes to impairment of immunity in pulmonary tuberculosis patients by alteration of butyrate and propionate producers," Environmental Microbiology, vol. 20, no. 1, pp. 402-419, 2018.

[93] M. F. Wipperman, D. W. Fitzgerald, M. A. J. Juste et al., "Antibiotic treatment for tuberculosis induces a profound dysbiosis of the microbiome that persists long after therapy is completed," Scientific Reports, vol. 7, no. 1, p. 10767, 2017.

[94] S. Namasivayam, M. Maiga, W. Yuan et al., "Longitudinal profiling reveals a persistent intestinal dysbiosis triggered by conventional anti-tuberculosis therapy," Microbiome, vol. 5, no. 1, p. 71, 2017.

[95] S. Negi, S. Pahari, H. Bashir, and J. N. Agrewala, "Gut microbiota regulates mincle mediated activation of lung dendritic cells to protect against Mycobacterium tuberculosis," Frontiers in Immunology, vol. 10, p. 1142, 2019.

[96] Y. Zhou, L. Chen, G. Sun, Y. Li, and R. Huang, "Alterations in the gut microbiota of patients with silica-induced pulmonary fibrosis," Journal of Occupational Medicine and Toxicology, vol. 14, no. 1, p. 5, 2019.

[97] B. Chacko, J. V. Peter, P. Tharyan, G. John, and L. Jeyaseelan, "Pressure-controlled versus volume-controlled ventilation for acute respiratory failure due to acute lung injury (ALI) or acute respiratory distress syndrome (ARDS)," The Cochrane Database of Systematic Reviews, vol. 1, no. 1, pp. CD008807-CD008807, 2010.

[98] M. L. Metersky and A. C. Kalil, "Management of ventilatorassociated pneumonia," Clinics in Chest Medicine, vol. 39, no. 4, pp. 797-808, 2018.

[99] R. P. Dickson, B. H. Singer, M. W. Newstead et al., "Enrichment of the lung microbiome with gut bacteria in sepsis and the acute respiratory distress syndrome," Nature Microbiology, vol. 1, no. 10, p. 16113, 2016.

[100] A. R. Panzer, S. V. Lynch, C. Langelier et al., "Lung microbiota is related to smoking status and to development of acute respiratory distress syndrome in critically ill trauma patients," American Journal of Respiratory and Critical Care Medicine, vol. 197, no. 5, pp. 621-631, 2018.

[101] R. Kapur, M. Kim, J. Rebetz et al., "Gastrointestinal microbiota contributes to the development of murine transfusionrelated acute lung injury," Blood Advances, vol. 2, no. 13, pp. 1651-1663, 2018.
[102] B. Li, G.-F. Yin, Y.-L. Wang, Y.-M. Tan, C.-L. Huang, and X.-M. Fan, "Impact of fecal microbiota transplantation on TGF- $\beta 1 /$ Smads/ERK signaling pathway of endotoxic acute lung injury in rats," 3 Biotech, vol. 10, no. 2, p. 52, 2020.

[103] J. U. Peled, S. M. Devlin, A. Staffas et al., "Intestinal microbiota and relapse after hematopoietic-cell transplantation," Journal of Clinical Oncology, vol. 35, no. 15, pp. 1650-1659, 2017.

[104] A. Mahmoodpoor, H. Hamishehkar, R. Asghari, R. Abri, K. Shadvar, and S. Sanaie, "Effect of a probiotic preparation on ventilator-associated pneumonia in critically ill patients admitted to the intensive care unit: a prospective doubleblind randomized controlled trial," Nutrition in Clinical Practice, vol. 34, no. 1, pp. 156-162, 2019.

[105] H. Weng, J.-G. Li, Z. Mao et al., "Probiotics for preventing ventilator-associated pneumonia in mechanically ventilated patients: a meta-analysis with trial sequential analysis," Frontiers in Pharmacology, vol. 8, p. 717, 2017.

[106] K.-j. Li, Z.-1. Chen, Y. Huang et al., "Dysbiosis of lower respiratory tract microbiome are associated with inflammation and microbial function variety," Respiratory Research, vol. 20, no. 1, pp. 272-272, 2019.

[107] D. Makrygiannakis, M. Hermansson, A. K. Ulfgren et al., "Smoking increases peptidylarginine deiminase 2 enzyme expression in human lungs and increases citrullination in BAL cells," Annals of the Rheumatic Diseases, vol. 67, no. 10, pp. 1488-1492, 2008.

[108] J. U. Scher, V. Joshua, A. Artacho et al., "The lung microbiota in early rheumatoid arthritis and autoimmunity," Microbiome, vol. 4, no. 1, p. 60, 2016.

[109] W. A. Vieira and E. Pretorius, "The impact of asthma on the gastrointestinal tract (GIT)," Journal of Asthma and Allergy, vol. 3, pp. 123-130, 2010.

[110] E. P. A. Rutten, K. Lenaerts, W. A. Buurman, and E. F. M. Wouters, "Disturbed intestinal integrity in patients with COPD: effects of activities of daily living," Chest, vol. 145, no. 2, pp. 245-252, 2014.

[111] L. N. Segal and M. J. Blaser, "A brave new world: the lung microbiota in an era of change," Annals of the American Thoracic Society, vol. 11, Supplement 1, pp. S21-S27, 2014.

[112] S. Anand and S. S. Mande, "Diet, microbiota and gut-lung connection," Frontiers in Microbiology, vol. 9, p. 2147, 2018.

[113] D. R. Samuelson, D. A. Welsh, and J. E. Shellito, "Regulation of lung immunity and host defense by the intestinal microbiota," Frontiers in Microbiology, vol. 6, p. 1085, 2015.

[114] T. Herbst, A. Sichelstiel, C. Schär et al., "Dysregulation of allergic airway inflammation in the absence of microbial colonization," American Journal of Respiratory and Critical Care Medicine, vol. 184, no. 2, pp. 198-205, 2011.

[115] R. L. Brown, R. P. Sequeira, and T. B. Clarke, "The microbiota protects against respiratory infection via GM-CSF signaling," Nature Communications, vol. 8, no. 1, p. 1512, 2017.

[116] M. H. Grayson, L. E. Camarda, S.-R. A. Hussain et al., "Intestinal microbiota disruption reduces regulatory $\mathrm{T}$ cells and increases respiratory viral infection mortality through increased IFN $\gamma$ production," Frontiers in Immunology, vol. 9, pp. 1587-1587, 2018.

[117] A. Cait, M. R. Hughes, F. Antignano et al., "Microbiomedriven allergic lung inflammation is ameliorated by shortchain fatty acids," Mucosal Immunology, vol. 11, no. 3, pp. 785-795, 2018. 
[118] X. Yang, H. Feng, X. Zhan et al., "Early-life vancomycin treatment promotes airway inflammation and impairs microbiome homeostasis," Aging, vol. 11, no. 7, pp. 2071-2081, 2019.

[119] A. J. Adami, S. J. Bracken, L. A. Guernsey et al., "Early-life antibiotics attenuate regulatory $\mathrm{T}$ cell generation and increase the severity of murine house dust mite-induced asthma," Pediatric Research, vol. 84, no. 3, pp. 426-434, 2018.

[120] S. L. Russell, M. J. Gold, L. A. Reynolds et al., "Perinatal antibiotic-induced shifts in gut microbiota have differential effects on inflammatory lung diseases," Journal of Allergy and Clinical Immunology, vol. 135, no. 1, pp. 100-109.e5, 2015.

[121] T. Olszak, D. An, S. Zeissig et al., "Microbial exposure during early life has persistent effects on natural killer T cell function," Science, vol. 336, no. 6080, pp. 489-493, 2012.

[122] J. C. Chen, C. C. Tsai, C. C. Hsieh, A. Lan, C. C. Huang, and S. F. Leu, "Multispecies probiotics combination prevents ovalbumin-induced airway hyperreactivity in mice," Allergologia et Immunopathologia, vol. 46, no. 4, pp. 354-360, 2018.

[123] V. M. B. Fonseca, T. M. S. Milani, R. Prado et al., "Oral administration of Saccharomyces cerevisiae UFMG A-905 prevents allergic asthma in mice," Respirology, vol. 22, no. 5, pp. 905-912, 2017.

[124] Z. Juan, S. Zhao-Ling, Z. Ming-Hua et al., "Oral administration of Clostridium butyricum CGMCC0313-1 reduces ovalbumin-induced allergic airway inflammation in mice," Respirology, vol. 22, no. 5, pp. 898-904, 2017.

[125] I. Spacova, M. I. Petrova, A. Fremau et al., "Intranasal administration of probiotic Lactobacillus rhamnosus GG prevents birch pollen-induced allergic asthma in a murine model," Allergy, vol. 74, no. 1, pp. 100-110, 2018.

[126] M.-H. Hsieh, R.-L. Jan, L. S.-H. Wu et al., "Lactobacillus gasseri attenuates allergic airway inflammation through PPAR $\gamma$ activation in dendritic cells," Journal of Molecular Medicine (Berlin, Germany), vol. 96, no. 1, pp. 39-51, 2018.

[127] H. Wang, J. X. Zhao, N. Hu, J. Ren, M. du, and M. J. Zhu, "Side-stream smoking reduces intestinal inflammation and increases expression of tight junction proteins," World Journal of Gastroenterology, vol. 18, no. 18, pp. 2180-2187, 2012.

[128] M. Reale, P. Boscolo, V. Bellante et al., "Daily intake of Lactobacillus casei Shirota increases natural killer cell activity in smokers," British Journal of Nutrition, vol. 108, no. 2, pp. 308-314, 2012.

[129] K. M. Antosca, D. A. Chernikova, C. E. Price et al., “Altered stool microbiota of infants with cystic fibrosis shows a reduction in genera associated with immune programming from birth," Journal of Bacteriology, vol. 201, no. 16, 2019.

[130] M. Bazett, M. E. Bergeron, and C. K. Haston, "Streptomycin treatment alters the intestinal microbiome, pulmonary $\mathrm{T}$ cell profile and airway hyperresponsiveness in a cystic fibrosis mouse model," Scientific Reports, vol. 6, no. 1, p. 19189, 2016.

[131] Q. F. Gui, H. F. Lu, C. X. Zhang, Z. R. Xu, and Y. H. Yang, "Well-balanced commensal microbiota contributes to anticancer response in a lung cancer mouse model," Genetics and Molecular Research, vol. 14, no. 2, pp. 5642-5651, 2015.

[132] S. Viaud, F. Saccheri, G. Mignot et al., "The intestinal microbiota modulates the anticancer immune effects of cyclophosphamide," Science, vol. 342, no. 6161, pp. 971-976, 2013.

[133] S. Gauguet, S. D'Ortona, K. Ahnger-Pier et al., "Intestinal microbiota of mice influences resistance to Staphylococcus aureus pneumonia," Infection and Immunity, vol. 83, no. 10, pp. 4003-4014, 2015.

[134] T. Ichinohe, I. K. Pang, Y. Kumamoto et al., "Microbiota regulates immune defense against respiratory tract influenza A virus infection," Proceedings of the National Academy of Sciences of the United States of America, vol. 108, no. 13, pp. 5354-5359, 2011.

[135] T. B. Tsay, M. C. Yang, P. H. Chen, C. M. Hsu, and L. W. Chen, "Gut flora enhance bacterial clearance in lung through toll-like receptors 4," Journal of Biomedical Science, vol. 18, no. 1, p. $68,2011$.

[136] L. W. Chen, P. H. Chen, and C. M. Hsu, "Commensal microflora contribute to host defense against Escherichia coli pneumonia through toll-like receptors," Shock, vol. 36, no. 1, pp. 67-75, 2011.

[137] C. T. Fagundes, F. A. Amaral, A. T. Vieira et al., "Transient TLR activation restores inflammatory response and ability to control pulmonary bacterial infection in germfree mice," Journal of Immunology, vol. 188, no. 3, pp. 1411-1420, 2012.

[138] K. Yoda, F. He, K. Miyazawa, M. Kawase, A. Kubota, and M. Hiramatsu, "Orally administered heat-killed Lactobacillus gasseri TMC0356 alters respiratory immune responses and intestinal microbiota of diet-induced obese mice," Journal of Applied Microbiology, vol. 113, no. 1, pp. 155-162, 2012.

[139] M. Mahooti, E. Abdolalipour, A. Salehzadeh, S. R. Mohebbi, A. Gorji, and A. Ghaemi, "Immunomodulatory and prophylactic effects of Bifidobacterium bifidum probiotic strain on influenza infection in mice," World Journal of Microbiology \& Biotechnology, vol. 35, no. 6, p. 91, 2019.

[140] T. Secher, I. Maillet, C. Mackowiak et al., "The probiotic strain Escherichia coli Nissle 1917 prevents papain-induced respiratory barrier injury and severe allergic inflammation in mice," Scientific Reports, vol. 8, no. 1, pp. 11245-11245, 2018.

[141] B. Zhang, J. An, T. Shimada, S. Liu, and K. Maeyama, "Oral administration of Enterococcus faecalis FK-23 suppresses Th17 cell development and attenuates allergic airway responses in mice," International Journal of Molecular Medicine, vol. 30, no. 2, pp. 248-254, 2012.

[142] M. Vareille-Delarbre, S. Miquel, S. Garcin et al., "Immunomodulatory effects of Lactobacillus plantarum on inflammatory response induced by Klebsiella pneumoniae," Infection and Immunity, vol. 87, no. 11, article e00570, 2019. 